\title{
Análisis de la productividad para las empresas certificadas y no certificadas en la Coalición Empresarial Anti-Contrabando (CEAC) en la ciudad de Cartagena, Colombia
}

\author{
Productivity analysis for certified and uncertified companies in the Business Ant \\ $i$-Smuggling Coalition (BASC) in the city of Cartagena, Colombia
}

Tomás José Fontalvo Herrera ${ }^{1}$

Recibido 14 de marzo de 2013, aceptado 22 de abril de 2015

Received: March 14, $2013 \quad$ Accepted: April 22, 2015

\begin{abstract}
RESUMEN
En éste artículo de investigación se evalúa la incidencia de la certificación CEAC en los indicadores de productividad para las empresas certificadas y no certificadas en Cartagena. En la metodología se calcularon los indicadores a 23 empresas, inicialmente el estudio se realizó durante el periodo 2008 y 2010 (empresas certificadas); posteriormente durante el 2010 (empresas certificadas y no certificadas). Se utilizó la técnica Análisis Discriminante; para explicar la pertenencia y discriminación, analizando los índices de productividad. De la función discriminante obtenida y los estadísticos analizados se puede concluir para el primer caso la certificación CEAC incide positivamente en la discriminación de los indicadores Utilidad Bruta/Valor Agregado (IP1), Utilidad Operacional/Capital Operativo (IP4) y Utilidad Neta/Capital Operativo (IP5) y el análisis de las medias no presentaron mejorías; para el segundo caso el indicador que presento diferencias significativas fue la Utilidad Operacional/Capital Operativo (IP4) no presentando mejorías en el análisis de las medias.
\end{abstract}

Palabras clave: Certificación, comercio seguro, productividad, sistema de gestión, análisis discriminante.

\begin{abstract}
In this article evaluates the incidence of BASC certification in productivity indicators for certified and non-certified companies in Cartagena. In the methodology indicators were calculated at 23 companies, initially the study was conducted during the period 2008 to 2010 (certified companies), later in 2010 (certified and non-certified companies). The technique used was discriminant analysis to explain the ownership and discrimination, analyzing productivity rates. From the discriminant function obtained and analyzed statistics can be concluded for the first case the BASC certification that the indicators positively affects discrimination in Gross Profit / Value Added (IP1) Operating Income / Operating Capital (IP4) and Net Income / Operating Capital (IP5) and the analysis of means present no improvement, for the second case the indicator that present significantly differences was the Operating Income / Operating Capital (IP4) showing no improvement in the analysis of means.
\end{abstract}

Keywords: Certification, sure trade, productivity, management system, discriminant analysis.

\footnotetext{
1 Universidad de Cartagena. Av. El consulado, Calle 30 № 48-152. Cartagena 1382. Cartagena de Indias, Colombia. E-mail: tfontalvoh@unicartagena.edu.co
} 


\section{INTRODUCCIÓN}

Hoy en día las transacciones comerciales a nivel nacional e internacional han sido afectadas por diferentes factores que afectan la seguridad y el comercio seguro, lo cual ha generado la necesidad de implementar normas Internacionales para mejorar esta situación, como lo es la Coalición Empresarial Anti-Contrabando (CEAC), estándar éste que busca mejorar los controles en la seguridad y de esta forma lograr que estos procesos de normalización redunden en la productividad de estas organizaciones. En consecuencia con lo anterior, en esta investigación se le dio respuesta a las siguientes preguntas. ¿Cuáles indicadores de productividad financiera permiten evaluar el desempeño de las entidades certificadas en CEAC? ¿Cuál es la diferencia en términos de productividad de las empresas de Cartagena certificadas y no certificadas, en la Coalición Empresarial Anti-Contrabando? ¿La certificación CEAC incide en el incremento de la productividad de las empresas certificadas en esta norma?

Para darle respuesta a las preguntas problemas, en esta investigación inicialmente se identificaron las empresas certificadas y no certificadas en CEAC, para posteriormente analizar los estados financieros de estas y seleccionar los rubros que permitieron calcular los indicadores de productividad de las empresas objeto de estudio, con lo cual se analizó qué tan significativas eran las diferencias entre este grupo de empresas utilizando el Análisis Discriminante. Y como consecuencia del análisis de los resultados de la Aplicación del Análisis Discriminante, evidenciar o no que tanto inciden los sistemas certificados CEAC en el mejoramiento de la productividad de las organizaciones.

En éste sentido, a nivel mundial se ha incrementado el comercio de bienes y servicios; y cada vez más las organizaciones empresariales se incorporan en las transacciones internacionales con los correspondientes requerimientos y los riesgos que implica dicha actividad [1-2]. Estos autores sostienen que mientras más compleja se configure la cadena de suministro de una empresa se vuelve mucho más vulnerable a las perturbaciones e interrupciones. De la misma forma se incrementan los requisitos que se deben cumplir para poder comercializar los productos en mercados extranjeros, la seguridad de los productos y de los consumidores finales es uno de ellos, por esto las organizaciones deben blindar sus operaciones de las actividades ilícitas que históricamente se han desarrollado paralelamente al comercio mundial de productos y servicios tal como lo sostiene [3]. Para prevenir este tipo de situaciones a nivel internacional diversas organizaciones donde incluso participan gobiernos han establecido una serie de estrategias estandarizadas para prevenir las interrupciones y problemas causados por actividades como el narcotráfico, el terrorismo y el contrabando con el fin de que las empresas que las implementen no se vean vinculadas a estas actividades y sean garantía para los consumidores de la seguridad de sus procesos y productos [4-5].

De igual forma procesos de normalización como la implementación del sistema de gestión BASC por sus siglas en ingles de "Business Anti-Smuggling Coalition" o CEAC por sus siglas en Español está orientada a estandarizar los procesos logísticos con el fin de mejorar la seguridad del producto y de los procesos mismos, se puede esperar entonces que la implementación de este sistema de gestión tenga implicaciones en la productividad de las organizaciones que la lleven a cabo, en otras palabras la certificación CEAC ayuda no solo a mejorar la seguridad en los procesos sino que también afecta de manera significativa otros aspectos de la organización tales como costos, agilidad, confianza del cliente, etc. Debido a lo anterior es importante dimensionar los efectos de éste tipo de certificaciones en las operaciones y resultados de las empresas en su conjunto [6-7].

Estos esfuerzos se ven reflejados en la implementación de estrategias que les permiten aumentar su productividad y prestar un mejor servicio a sus clientes y consumidores [8].

De ahí la importancia de establecer relaciones de colaboración con el fin de regular las actividades logísticas y ponerle freno a todas las actividades externas que puedan afectar la normalidad de las actividades de la organización, sin embargo el aumento de los miembros participantes en la cadena puede generar también debilidades y vulnerabilidades que es preciso identificar y tratar de subsanar; de lo anterior se concluye que la clave para garantizar la seguridad en el comercio se encuentra en estandarizar la seguridad en los procesos desarrollados por cada uno de los participantes para que cumplan con tal fin [9-10]. 
Las actividades ilícitas mencionadas anteriormente pueden afectar cualquier tipo de empresa independientemente de las actividades a las cuales estas se dediquen; por lo cual se debe garantizar la seguridad de los productos desde el mismo momento en que se empieza a configurar la red logística, una vez más se insiste sobre la necesidad de establecer estrategias de colaboración entre cada uno de los participantes de la cadena de suministro para poder alcanzar el logro de un comercio seguro. De igual manera, esto permite optimizar los costos para lograr una producción y distribución segura para que las operaciones contribuyan con el logro de un mejor desempeño en el ámbito operativo y financiero de la organización y mejorar la confianza de los grupos de interés, con lo que se pueda obtener nuevos clientes y posicionamiento en el mercado, lo que se traduzca en el bienestar social y económico de la empresa.

\section{ANÁLISIS TEÓRICO}

Con la certificación CEAC las empresas pueden también alcanzar mayor participación en las transacciones comerciales de orden internacional. Éste sistema de gestión en control y seguridad nace como el reto organizacional consistente en una mayor colaboración entre los participantes de la cadena para hacer frente a problemas comunes y que afectan a todos [11].

Al certificar en CEAC la organización que asume el estándar se convierte en un operador económico autorizado; este nombre se le otorga a las organizaciones vinculadas al comercio internacional y que dan garantía de confianza y seguridad en sus operaciones.

El sistema de gestión CEAC es una forma de garantizar la seguridad del producto y su trazabilidad; es decir mediante esta estrategia se puede alcanzar un mayor nivel en la calidad de los servicios y productos a la vez que se incurren en menos costos lo que puede implicar un paso hacia la planeación colaborativa, el rediseño de los procesos y la innovación organizacional [12-13].

Medina [14], señala que existe la necesidad dentro de las empresas vinculadas a las exportaciones de incrementar su productividad modernizando sus sistemas de gestión y la tecnología que utilizan, la certificación CEAC constituye una alternativa para lograrlo.

\section{Indicadores de productividad}

En éste artículo de investigación se presentan 2 análisis para evaluar las empresas objeto de esta investigación, en el primer caso se estudian los indicadores de productividad de las empresas certificadas durante el 2008 y 2010 y el segundo los indicadores de las empresas certificadas y no certificadas que presentaron sus estados financieros en el 2010. Se utilizan los indicadores de productividad que se presentan en la Tabla 1, teniendo en cuenta que estos permiten evaluar de forma puntual la creación de valor y riquezas al interior de las organizaciones dinamizando no sólo los recursos en la organización como tal, sino también en el mercado [15].

Martínez [16], sostiene que los indicadores operacionales junto con los financieros cada vez demuestran su importancia y utilidad al momento de la toma de decisiones, sin embargo Bortesi [17] afirma que también existen unos aspectos que no necesariamente pueden calificarse cuantitativamente sino que de manera intrínseca contribuyen al desempeño productivo de las empresas. Por lo general el incremento de la productividad se relaciona también con un aumento en la calidad de los productos, pero Rincón [18] sostiene que el aumento en la productividad puede conllevar a una disminución en la calidad si no se controlan los procesos. Un buen desempeño de la productividad empresarial puede ser producto de la innovación, en este sentido son varias las fuentes de innovación empresarial entre las que se puede identificar las tecnologías de la información, el rediseño organizativo y la capacitación, los anteriores elementos son brindados e implementados por el Sistema de Gestión CEAC [19].

\section{De la certificación CEAC sobre la productividad mediante el análisis discriminante}

El sistema de gestión CEAC está relacionado con elementos como la infraestructura de la empresa, la forma de trabajo, la innovación y la investigación, los cuales afectan significativamente el desempeño de la productividad [19-22], de ahí la necesidad de evaluar la incidencia de la certificación CEAC en la productividad de las empresas. 
Así mismo, Vivanco, Martinez y Taddei [23], hacen uso de la metodología que proporciona el Análisis Discriminante ADM con el propósito de estudiar y analizar los niveles de competitividad en diferentes organizaciones. El Análisis Discriminante tiene en cuenta el comportamiento sistémico de las organizaciones e intenta identificar las variables que mejor describen el comportamiento del sistema; a través de ciertas herramientas que valoran la similitud o diferencia entre las mismas [24-25].

Los planteamientos de los autores previos, muestran la importancia que han tenido la aplicación del ADM para estudiar y analizar la competitividad en las organizaciones. En este mismo sentido es importante señalar que estos sistemas normalizados y estandarizados bajo el estándar CEAC, permiten consolidar procesos e eliminan toda posibilidad de situación o condiciones de riesgo o inseguridad en los procesos de intercambios de mercancía a nivel nacional e internacionalmente, lo cual se refleja de manera directa en los estados financieros y en los rubros analizados relacionados con las empresas que implementen este modelo. Lo que a la postré también incidirá en el bienestar social de todos los grupos de interés que aporten y participen en los procesos de normalización CEAC.

Cabe señalar que una mejora en los niveles de productividad está asociada con un incremento en la generación de valor agregado, en la Utilidad Bruta, Utilidad Operacional y Utilidad Neta como se puede ver en los indicadores de productividad y en el denominador de los indicadores que se presentan en la Tabla 1. Por lo tanto, hablar de generación de valor y productividad está asociado a generar riqueza y a llenar necesidades de los diferentes actores y grupos de interés donde intervienen las organizaciones certificadas, lo que a la postre se transforma en un incremento del bienestar social y económico de las empresas que implementan este tipo de estándares y de los diferentes actores que interactúan con las organizaciones que alcanzan la certificación la Coalición Empresarial AntiContrabando (CEAC).

\section{METODOLOGÍA}

Esta investigación se abordó bajo una concepción de la realidad holista analizando la unidad y la diversidad de dos grupos de empresas certificadas y no certificadas en la Coalición Empresarial Anti-Contrabando (CEAC). De igual manera el estado de la realidad analizada se entendió como dialectico y complejo toda vez que se confrontaron y compararon dos grandes grupos de empresas con características distintas en cuanto a la normalización de sus procesos y resultados de productividad bajo criterios de seguridad en el comercio. Así mismo la concepción de la realidad de las organizaciones en esta investigación se consideró como empresa cerrada y abierta a la vez, teniendo en cuenta la naturaleza propia de estas, asociada con sus actividades comerciales y teniendo en cuenta que la normalización se hace a nivel interno y los procesos de intercambio de mercancías se realiza a nivel nacional e Internacional, es decir externo.

Como principios que definen la articulación o vinculación entre los componentes o estructuras de la realidad estudiada se analizó la explicación multicausal, considerando que se estudió como la certificación en CEAC incide o no positivamente en el mejoramiento de una serie de variables, rubros financieros e indicadores con la productividad de las empresas certificadas.

La esencia de la ciencia en esta investigación se generó como resultado de un enfoque empirista y racionalista toda vez que la verdad se generó del estudio del objeto, es decir de una serie de variables e indicadores relacionadas con las empresas certificadas y no certificadas en CEAC objeto de la investigación. Estudiadas a través de un análisis racional complementario del sujeto investigador al elaborar una serie de construcciones y análisis del objeto de la investigación, es decir de las empresas certificadas y no certificadas en CEAC.

La concepción de verdad en esta investigación se abordó desde un enfoque empirista considerando que se utilizó la verificación estadística y probabilística de una población y muestra para realizar inferencias soportadas en criterios de validez estadísticos por medio del análisis de parámetros e indicadores de productividad utilizando el Análisis Discriminante ADM.

También se consideró un enfoque racionalista por medio del cual se construyó y argumentó de manera cualitativa y cuantitativa el planteamiento y la relación entre las variables para el estudio. Se 
triangulo la información cualitativa y cuantitativa relacionada con los indicadores seleccionados y los resultados arrojados por el Análisis Discriminante, para realizar el análisis y las conclusiones de esta investigación que sustentaron la confiabilidad de los resultados alcanzados.

La lógica del método de ésta investigación fue mixto toda vez que se realizó un análisis inductivo al analizar las variables, indicadores y particularidades de un grupo de empresas certificadas y no certificadas en CEAC. Y deductivo toda vez que producto del análisis racionalista se pudo llegar a conclusiones generales soportadas en criterios de confiabilidad y validez estadística.

Para lo anterior, se identificaron las variables, rubros financieros e indicadores de productividad desde una perspectiva cualitativa y estadística, para posteriormente utilizar el Análisis Discriminante Multivariado (ADM) y de esta forma estudiar los indicadores seleccionados de las empresas en los periodos 2008 y 2010 y poder evidenciar o no la incidencia de la normalización CEAC en la productividad de las empresas de la ciudad de Cartagena. Para este trabajo se utilizaron los indicadores de productividad que se presentan en la Tabla 1.

Tabla 1. Indicadores de productividad ${ }^{2}$.

\begin{tabular}{|l|c|}
\hline IP1 & Ecuación \\
\hline IP2 & $\frac{\text { Utilidad Bruta }}{\text { Valor agregado (ventas }- \text { pagos a proveedores }+ \text { inventarios })} \times 100$ \\
\hline IP3 & $\frac{\text { Utilidad Operacional }}{\text { Valor agregado }(\text { ventas }- \text { pagos a proveedores }+ \text { inventarios })} \times 100$ \\
\hline IP4 & $\frac{\text { Utilidad Neta }}{\text { Valor agregado (ventas }- \text { pagos a proveedores }+ \text { inventarios })} \times 100$ \\
\hline IP5 & $\frac{\text { Utilidad Operacional }}{\text { Capital Operativo (activos corrientes y fijo })} \times 100$ \\
\hline
\end{tabular}

2 El valor agregado, es el valor creado durante la prestación del servicio o la realización del producto. La utilidad Bruta se entiende en esta investigación como los ingresos producto de las ventas de bienes y servicios, menos los costos para producir estos. La utilidad Operacional, se entiende como el resultado de tomar los ingresos de la operación menos los costos y gastos de la operación. La Utilidad Neta, es la utilidad que finalmente se reparte entre los socios de las empresas certificadas en CEAC.
Para analizar los indicadores por medio de ADM de las empresas de la ciudad de Cartagena, se utilizó la distancia $\mathrm{D}^{2}$ de Mahalanobis. La relevancia de la distancia de Mahalanobis se centra en que por su aplicación se determina la diferencia o igualdad entre dos variables considerando la correlación entre cada una de ellas y permite saber cuáles son las variables o indicadores con mayor variación [26].

Como cualquier otra técnica estadística paramétrica se verificó el cumplimiento de los supuestos. El Análisis Discriminante se apoyó en los siguientes supuestos:

- Normalidad Multivariante.

- Igualdad de Matrices de Varianza-Covarianza.

- Linealidad.

- Ausencia de multicolinealidad y singularidad.

\section{Muestra}

Para este estudio se tomó como población las empresas certificadas de la ciudad de Cartagena y como muestra un total de 23 empresas certificadas de la ciudad de Cartagena. Las cuales se compararon con 23 empresas no certificadas en CEAC.

\section{Fuentes y datos}

Para la realización del trabajo se tomó como fuente la información registrada en el Organismo Internacional CEAC y en la Superintendencia Financiera de Colombia en los años 2008 y 2010, de donde se extrajeron los datos y variables asociados con los rubros requeridos para calcular los indicadores de productividad objeto de esta investigación.

\section{RESULTADOS}

Para el estudio de las empresas de Cartagena certificadas y no certificadas en CEAC se revisaron los criterios que se presentan a continuación:

\section{Verificación de los supuestos}

En primer lugar se verificaron los supuestos del modelo, en la Tabla 2 y Tabla 3 se presentan los resultados para ambos casos:

1 caso empresas certificadas 2008 y 2010

2 caso empresas certificadas y no certificadas 2010

Autores [27-28], afirma la importancia de tomar muestras mayores a 20 para que el modelo generado 
Tabla 2. Prueba de Shapiro \& Wilk para la comprobación de la normalidad de los indicadores de productividad del año 2008 y 2010 empresas certificadas respectivamente.

\begin{tabular}{|c|c|c|c|c|c|c|}
\hline \multirow{2}{*}{} & \multicolumn{2}{|c|}{ Shapiro-Wilk 2008 } & \multicolumn{3}{c|}{ Shapiro-Wilk 2010 } \\
\cline { 2 - 7 } & Estadístico & gl & Sig. & Estadístico & gl & Sig. \\
\hline IP1 & 0,901 & 23 & 0.213 & 0,857 & 23 & 0,299 \\
\hline IP2 & 0,811 & 23 & 0,101 & 0,912 & 23 & 0,144 \\
\hline IP3 & 0,893 & 23 & 0,118 & 0,261 & 23 & 0,88 \\
\hline IP4 & 0,928 & 23 & 0,111 & 0,921 & 23 & 0,172 \\
\hline IP5 & 0,954 & 23 & 0,351 & 0,791 & 23 & 0,21 \\
\hline
\end{tabular}

Tabla 3. Prueba de Shapiro \& Wilk para la comprobación de la normalidad de los indicadores de productividad del año 2010 de las empresas no certificadas.

\begin{tabular}{|c|c|c|c|}
\hline \multirow{2}{*}{} & \multicolumn{3}{|c|}{ Shapiro-Wilk 2010 } \\
\cline { 2 - 4 } & Estadístico & gl & Sig. \\
\hline IP1 & 0,644 & 23 & 0.190 \\
\hline IP2 & 0,779 & 23 & 0.199 \\
\hline IP3 & 0,973 & 23 & 0,757 \\
\hline IP4 & 0,95 & 23 & 0,294 \\
\hline IP5 & 0,955 & 23 & 0,371 \\
\hline
\end{tabular}

sea confiable y valido, lo cual se consideró en este trabajo de investigación teniendo en cuenta que se tomaron 23 muestras para ambos casos, por lo que el modelo es robusto de acuerdo a las especificaciones de tamaño sugerido.

\section{Homogeneidad de matrices de varianza-covarianza}

El supuesto de igualdad de matrices de varianzacovarianza para el primer caso empresas certificadas durante los periodos 2008 y 2010 y segundo caso empresas certificadas y no certificadas periodo 2010, se comprobó con la prueba de Box, como se muestra en la Tabla 4 y 5.

Los resultados obtenidos para el primer caso, del estadístico de contraste $\mathrm{M}=42,557$ y un valor de $\mathrm{F}=$ 2,488 con una probabilidad asociada $\mathrm{p}=0,001$, de donde se deduce que la capacidad para evidenciar diferencias entre los dos grupos de empresas en buena.
Tabla 4. Resultados de la prueba de BOX empresas certificadas periodos 2008 y 2010.

\begin{tabular}{|c|r|r|}
\hline \multicolumn{2}{|c|}{ M de Box } & 42,557 \\
\hline \multirow{3}{*}{ F } & Aprox. & 2,488 \\
\cline { 2 - 3 } & gl1 & 15 \\
\cline { 2 - 3 } & gl2 & 7794,947 \\
\cline { 2 - 3 } & Sig. & 0,001 \\
\hline
\end{tabular}

Tabla 5. Resultados de la prueba de BOX empresas certificadas y no certificadas periodo 2010 .

\begin{tabular}{|l|c|r|}
\hline \multicolumn{2}{|l|}{ M de Box } & 119,242 \\
\hline F & Aprox. & 6,947 \\
\cline { 2 - 3 } & gl1 & 15 \\
\cline { 2 - 3 } & gl2 & 7410,315 \\
\cline { 2 - 3 } & Sig. & 0 \\
\hline
\end{tabular}

Asimismo, los resultados obtenidos para el segundo caso, del estadístico de contraste $\mathrm{M}=119,242$ y un valor de $\mathrm{F}=6,947$ con una probabilidad asociada $\mathrm{p}=0$, de estos resultados se demuestra que la capacidad de separación de las 2 poblaciones de empresas estudiadas es aceptable.

\section{Linealidad y multicolinealidad y singularidad}

Los supuestos de linealidad y multicolinealidad y singularidad no serán revisados, teniendo en cuenta que para la elaboración del modelo establecido se aplicó el método paso a paso.

\section{Selección de las variables que mejor discriminan}

Con el fin de identificar los indicadores que muestran discriminación entre las poblaciones de las empresas certificadas y no certificadas en CEAC, se calculó la distancia $\mathrm{D}^{2}$ de Mahalanobis y el Lambda Wilks en estos dos grupos. Una vez utilizado la técnica Análisis Discriminante se construyeron los coeficientes para la elaboración de las funciones del modelo que se muestran en Tabla 6 y Tabla 7 para ambos casos donde se resaltan los indicadores que presentaron diferencias significativas y en las ecuaciones (1), (2), (3) y (4).

$\mathbf{Z}_{\mathbf{1}}=\mathrm{IP} 1 *(5,007)+\mathrm{IP} 2 *(-2,253)+\mathrm{IP} 3 *(-3,923)$

+ IP4* 4 (7,117) + IP5*(-0,601)-(1,479)

$\mathbf{Z}_{\mathbf{2}}=$ IP1 $*(5,721)+\operatorname{IP} 2 *(-4,265)+\operatorname{IP} 3 *(-8,877)$

+ IP4*(7,924) + IP5*(1,454)-(1,646) 
$\mathbf{Z}_{\mathbf{3}}=\mathrm{IP} 1 *(5,509)+\mathrm{IP} 2 *(-4,275)+\mathrm{IP} 3 *(1,068)$

+ IP4*(11,084) + IP5*(-9,754)-(1,698)

$\mathbf{Z}_{\mathbf{4}}=\mathrm{IP} 1 *(5,007)+\mathrm{IP} 2 *(-10,29)+\mathrm{IP} 3 *(0,604)$

+ IP4*(15,755) + IP5*(-10,701)-(1,567)

Tabla 6. Coeficientes de la función de clasificación empresas certificadas 2008 y 2010.

\begin{tabular}{|l|c|c|}
\hline \multirow{2}{*}{} & \multicolumn{2}{|c|}{ AÑ } \\
\cline { 2 - 3 } & $\mathbf{2 0 0 8}$ & $\mathbf{2 0 1 0}$ \\
\hline IP1 & 5,007 & 5,721 \\
\hline IP2 & $-2,253$ & $-4,265$ \\
\hline IP3 & $-3,923$ & $-8,877$ \\
\hline IP4 & 7,117 & 7,924 \\
\hline IP5 & $-0,601$ & 1,454 \\
\hline (Constante) & $-1,479$ & $-1,646$ \\
\hline
\end{tabular}

Tabla 7. Coeficientes de la función de clasificación empresas certificadas y no certificadas 2010.

\begin{tabular}{|c|c|c|}
\hline \multirow{2}{*}{} & \multicolumn{2}{|c|}{$\mathbf{2 0 1 0}$} \\
\cline { 2 - 3 } & Certificadas & No certificadas \\
\hline IP1 & 5,509 & 5,007 \\
\hline IP2 & $-4,275$ & $-10,29$ \\
\hline IP3 & 1,068 & 0,604 \\
\hline IP4 & 11,084 & 15,755 \\
\hline IP5 & $-9,754$ & $-10,701$ \\
\hline (Constante) & $-1,698$ & $-1,567$ \\
\hline
\end{tabular}

Como resultado de la aplicación del Análisis Discriminante se puede afirmar que los Indicadores de productividad para el primer caso de empresas certificadas en CEAC, estudiado presentan diferencias significativas, generando como resultado un error Tipo I de 21,7\%, un error Tipo II de 69,6\% para una eficacia de clasificación promedio de $54,3 \%$, como se muestra en Tabla 8.

Obtener un $54,3 \%$ es coherente y pertinente con el hecho de que solo el indicador IP4 presentó diferencias significativas una vez aplicado el Análisis Discriminante.

Asimismo, del segundo caso estudiado se puede observar que también presentan diferencias significativas, generando como resultado un error
Tipo I de 56,6\%, un error Tipo II de 18,2\% para una efectividad de clasificación de $62,2 \%$, como se muestra en Tabla 9.

Tabla 8. Resultados de la clasificación empresas certificadas 2008 y 2010.

\begin{tabular}{|c|c|c|c|c|c|}
\hline & & \multirow[t]{2}{*}{ Año } & \multicolumn{2}{|c|}{$\begin{array}{c}\text { Grupo de } \\
\text { pertenencia } \\
\text { pronosticado }\end{array}$} & \multirow[t]{2}{*}{ Total } \\
\hline & & & 2008 & 2010 & \\
\hline \multirow[t]{4}{*}{ Original } & \multirow[t]{2}{*}{ Recuento } & 2008 & 18 & 5 & 23 \\
\hline & & 2010 & 16 & 7 & 23 \\
\hline & \multirow[t]{2}{*}{$\%$} & 2008 & 78,3 & 21,7 & 100,0 \\
\hline & & 2010 & 69,6 & 30,4 & 100,0 \\
\hline
\end{tabular}

Tabla 9. Resultados de la clasificación empresas certificadas y no certificadas 2010 .

\begin{tabular}{|c|c|c|c|c|}
\hline \multirow{4}{*}{ Año } & \multicolumn{2}{|c|}{$\begin{array}{c}\text { Grupo de pertenencia } \\
\text { pronosticado }\end{array}$} & \multirow{2}{*}{ Total } \\
\cline { 3 - 5 } & & Certificadas & $\begin{array}{c}\text { No } \\
\text { certificadas }\end{array}$ & \\
\hline \multirow{4}{*}{ Recuento } & Certificadas & 10 & 13 & 23 \\
\cline { 2 - 5 } & No certificadas & 4 & 18 & 22 \\
\cline { 2 - 5 } & $\begin{array}{c}\text { Casos } \\
\text { desagrupados }\end{array}$ & 0 & 1 & 1 \\
\hline \multirow{4}{*}{$\%$} & Certificadas & 43,5 & 56,5 & 100 \\
\cline { 2 - 5 } & No certificadas & 18,2 & 81,8 & 100 \\
\cline { 2 - 5 } & $\begin{array}{c}\text { Casos } \\
\text { desagrupados }\end{array}$ &, 0 & 100 & 100 \\
\hline
\end{tabular}

Evaluación de la productividad en las empresas de la ciudad de Cartagena

Al revisar las medias en los indicadores de productividad de las empresas de Cartagena objeto del trabajo se observa que para el primer caso las empresas certificadas 2008 y 2010 no presentan mejorías y para el segundo caso empresas certificadas y no certificadas 2010 las medias tampoco presentan mejorías como se muestra en la Tabla 10 y Tabla 11. Es decir a pesar de que existen diferencias significativas en las poblaciones estudiadas para los casos estudiados por ADM. No se observa una incidencia de la certificación CEAC, en el incremento de la Utilidad Neta, Utilidad Bruta y Utilidad Operacional (variables propias de los indicadores establecidos para esta investigación).

Del análisis Discriminante se puede afirmar también que las poblaciones estudiadas de las 
empresas certificadas y no certificadas, presentan diferencias significativas, lo que sugiere que poseen características distintas producto de la certificación CEAC, sin embargo cuando revisamos el comportamiento de las medias de los indicadores financieros presentados en la Tabla 1 de las empresas certificadas en los años 2008 y 2010, se puede analizar que no existen una evolución o mejora en el comportamiento de los indicadores de productividad seleccionados para esta investigación. Por lo que sería importante para futuras investigaciones aplicar esta misma metodología con otro tipo de indicadores de productividad de manera que se pueda analizar la causalidad o multicausalidad de origen que genera las diferencias significativas cuando se implementa el modelo de Coalición Empresarial Anti-Contrabando CEAC.

Tabla 10. Medias de los indicadores financieros empresas certificadas 2008 y 2010.

\begin{tabular}{|c|c|c|c|}
\hline \multicolumn{2}{|c|}{ Año } & Media & Desv. típ. \\
\hline \multirow{4}{*}{2008} & IP1 &, 2659 &, 27115 \\
\cline { 2 - 4 } & IP2 &, 0440 &, 14579 \\
\cline { 2 - 4 } & IP3 &, 0491 &, 10371 \\
\cline { 2 - 4 } & IP4 &, 0800 &, 14143 \\
\cline { 2 - 4 } & IP5 &, 0625 &, 10450 \\
\hline \multirow{4}{*}{2010} & IP1 &, 2552 &, 27324 \\
\cline { 2 - 4 } & IP2 &,- 0022 &, 19227 \\
\cline { 2 - 4 } & IP3 &, 0052 &, 14857 \\
\cline { 2 - 4 } & IP4 &, 0539 &, 17432 \\
\cline { 2 - 4 } & IP5 &, 0505 &, 11765 \\
\hline
\end{tabular}

Tabla 11. Medias de los indicadores financieros empresas certificadas y no certificadas 2010.

\begin{tabular}{|c|c|c|c|}
\hline \multicolumn{2}{|c|}{ Año } & Media & Desv. típ. \\
\hline \multirow{4}{*}{ Certificadas } & IP1 &, 3155 &, 24456 \\
\cline { 2 - 4 } & IP2 &, 0641 &, 12654 \\
\cline { 2 - 4 } & IP3 &, 2461 &, 98583 \\
\cline { 2 - 4 } & IP4 &, 0746 &, 11696 \\
\cline { 2 - 4 } & IP5 &, 0557 &, 07458 \\
\hline \multirow{4}{*}{$\begin{array}{c}\text { No } \\
\text { certificadas }\end{array}$} & IP1 &, 2599 &, 27871 \\
\cline { 2 - 4 } & IP2 &,- 0060 &, 19592 \\
\cline { 2 - 4 } & IP3 &, 0039 &, 15192 \\
\cline { 2 - 4 } & IP4 &, 0496 &, 17716 \\
\cline { 2 - 4 } & IP5 &, 0373 &, 13367 \\
\hline
\end{tabular}

\section{DISCUSIÓN}

$\mathrm{Al}$ analizar las funciones discriminantes generadas en la Tabla 6 y Tabla 7 se puede concluir para el primer caso, es decir empresas certificadas 2008 y 2010 que los indicadores como (IP1), (IP4) y (IP5) discriminan bien mejorando de un periodo a otro, es decir presentan diferencias de un año al otro y para el segundo caso empresas certificadas y no certificadas el indicador (IP4) fue el único indicador que presento mejorías en el análisis de la discriminación. Lo que no sucedió para el resto de indicadores objeto de esta investigación.

Al revisar las medias de los indicadores que se presentan en la Tabla 10 y Tabla 11 se percibe que estas no mejoraron. Es importante señalar que el valor agregado y el capital operativo están relacionados con los procesos operativos y por lo tanto están asociados también a la productividad de la firma. Los resultados obtenidos en esta investigación concuerdan con metodologías y análisis realizados anteriormente en los cuales se evalúa la productividad, mediante indicadores y se obtienen resultados que evidencian diferencias significativas entre grupos de empresas [29-30].

Así mismo, investigaciones desarrolladas por Fontalvo, Mendoza y Morelos [31] y Fontalvo, Mórelos y De la Hoz [45], plantean que lo sistemas normalizados generan características diferenciales cuando se implementan en estos y adicionalmente inciden en los estados financieros de las empresas. De la misma forma se destaca que mediante la metodología desarrollada en esta investigación se establecen alternativas soportadas en calculo multivariable para la evaluación del desempeño de la productividad, diferentes a las desarrolladas por Ballesteros y Ballesteros [32] y Gómez [33] que ofrecen confiabilidad y resultados efectivos.

Por otra parte, los resultados obtenidos en ésta investigación son coherentes con los planteamientos de Avendaño y Varela [32] quienes plantean que la normalización e implementación de estándares genera condiciones distintivas en la competitividad de las organizaciones que los implementan. Lo cual se demostró con los resultados arrojados por ADM.

\section{CONCLUSIONES}

En ésta investigación se analizó, estudió e interpretó los resultados asociados a 2 grupos empresariales 
conformados por 23 empresas de la ciudad de Cartagena certificadas en CEAC, el modelo del primer caso de empresas certificadas 2008 y 2010 presentó una efectividad aceptable de $54,3 \%$ y para el 2 caso, es decir las empresas certificadas y no certificadas en CEAC, el modelo presento una buena clasificación de $62,2 \%$, de donde se deduce una capacidad aceptable para analizar qué tan diferentes son los indicadores de estos grupos empresariales, específicamente para los indicadores (IP1), (IP4) y (IP5) del primer caso y (IP4) para el segundo caso.

A pesar de que se encontró que hay diferencias significativas en las poblaciones para el primer caso y segundo caso analizado, no existe evidencia estadística por medio de las medias calculadas, de que la normalización en CEAC incidió a favor, en los resultados asociados al cálculo de los indicadores de productividad. Sin embargo la certificación CEAC puede incidir de manera positiva en otros contextos cuando se analicen otros indicadores de productividad, considerando que se pudo observar del ADM que los grupos empresariales producto de la certificación si poseen elementos diferenciadores.

Como continuación de este trabajo de investigación, se hace necesario analizar las diferentes variables, procesos y requisitos del estándar CEAC mirar su causalidad y multicausalidad con los rubros financieros y su relación con los indicadores de productividad a diseñar y evaluar. Y poder utilizar estos para calcular otros indicadores de productividad, para estudios similares. Además, aplicar la metodología planteada en este trabajo investigativo para confrontar y verificar no solo las diferencias significativas que existen en los grupos empresariales que implementan estos modelos o no, sino estudiar, comprender e interpretar la incidencia y el grado en que afectan estos sistemas normalizados de comercio seguro CEAC en el mejoramiento del comportamiento de los indicadores de productividad financiera seleccionados. De tal forma que se pueda priorizar sobre que variables y procesos estandarizados del comercio seguro hay que establecer procesos de planificación, control y seguimiento, con lo que se pueda incrementar la productividad financiera y por ende aumentar el bienestar social y económico de todos los grupos de interés que intervienen en la cadena logística de este tipo de empresas que se caracterizan por importar y exportar.
Como prolongación de éste trabajo también se podrá analizar la incidencia de esta norma internacional u otras normas internacionales sobre diversos tipos de indicadores como son los de productividad, eficiencia y seguridad, para analizar la efectividad que tiene en otros grupos empresariales que importan en Colombia o a nivel internacional. Lo que le permitirá a la alta dirección de estos grupos empresariales redireccionar las acciones y poder evaluar de manera efectiva la aplicación o no de la norma CEAC o cualquier norma implementada.

\section{REFERENCIAS}

[1] R. Young y P. Esqueda. "Vulnerabilidades de la Cadena de Suministros: Consideraciones para el caso de América Latina". Revista Latinoaméricana de Administración. Vol. $34 \mathrm{~N}^{\circ}$ 7, pp. 63-78. Enero 2005. ISSN: 1012-8255.

[2] O. Valdevira, E. Díaz y R. Sans "Impacto y situación de los operadores de carga terrestre de mercancías en relación a la normativa actual en materia de gestión de la seguridad en la cadena de suministro, como paso para obtener el estatus de operador seguro". Estudios de Construcción y transporte. Vol. $111 \mathrm{~N}^{\circ}$ 2, pp. 65-73. Diciembre 2009. ISSN: 1020-1017.

[3] M. Perusset. "Conductas y procedimientos fuera de la ley: comercio ilícito, líderes y prácticas". Universitas Humanística. Vol. 63 No 1, pp. 203-209. Diciembre 2007. ISSN: 0120-4807.

[4] A. Correa y R. Gómez. "Seguridad en la Cadena de Suministro basado en la norma ISO 28001 para el sector y sus derivados como estrategia para su competitividad". Boletín de ciencias de la tierra. Vol. $28 \mathrm{~N}^{\mathrm{o}}$ 7, pp. 39-50. Diciembre 2010. ISSN: 1888-4423.

[5] A. Maruchek, N. Greis, C. Mena y L. Cai. "Product safety and security in the global supply chain: issues, challenges and research opportunities". Journal Of Operation Management. Vol. $29 \mathrm{~N}^{\mathrm{o}}$ 2, pp. 707-720. Marzo 2011. ISSN: 0164-1212.

[6] B. Correa. "Sistema de gestión en control y seguridad BASC para Pymes en Medellín y área metropolitana". Universidad Católica Popular de Risaralda, Colombia. No 30 , pp. 1-10. Abril 2009. 
[7] T. Iturralde, A. Maseda y L. Ruiz. "La gestión de la liquidez: necesidades y excedentes de tesorería". Boletín económico de ICE. Vol. $2384 \mathrm{~N}^{\circ} 1$, pp. 29-41. Octubre 2005. ISSN: 0214-8307.

[8] J. Fariñas. "Comercio Internacional, Empresa y Competitividad". Cuadernos Económicos de ICE. Vol. $82 \mathrm{~N}^{\circ}$ 5, pp. 5-10. Septiembre 2011. ISSN: 0210-2633.

[9] H. Pfohl, H. Kölher and T. David. "State of the art in Supply Chain Management Risk research: Empirical and conceptual findings and a roadmap for the implementation in practice". Logistic Research. Vol. $2 \mathrm{~N}^{\mathrm{o}} 1$, pp. 33-44. Junio 2010. ISSN: 0120-4807.

[10] I. Manuj y J. Mentzer. "Global Supply Chain Risk Management Strategies". International Jornal of Physical Distribution and Logistic Management (abstract). Vol. $8 \mathrm{~N}^{\circ}$ 2, pp. 192223. Noviembre 2008. ISSN: 0960-0035.

[11] A. Osorio. "Impacto de las certificaciones ISO 9000 y BASC en los despachos de exportación definitiva de las agencias de aduana peruanas 2004-2008". Revista de Ciencias Empresariales de la Universidad de San Martin de Porres. Vol. $1 N^{\circ}$ 2, pp. 25-38. Diciembre 2010. ISSN: 0716-1042.

[12] Aecaf, Asociación Española de Carga Fraccionada. "Impacto y situación de los operadores de carga terrestre de mercancías en relación a la normativa actual en materia de gestión de la seguridad en la cadena de suministro como paso para obtener el estatus de operado seguro". Ministerio de Fomento. Julio 2008.

[13] H. Lee y S. Whang. "Higher Supply Chain Security with lower cost: Lesson for Total Quality Management". International Journal of Production Economics. Vol. $86 \mathrm{~N}^{\mathrm{o}} 1$, pp. 289-300. Junio 2003. ISSN: 0718-1876.

[14] J. Medina. "Modelo integral de productividad: aspectos importantes para su implementación". Revista Escuela de Administración y de negocios. Vol. $69 \mathrm{~N}^{\circ}$ 1, pp. 110-119. Septiembre 2010. ISSN: 0120-8160.

[15] J. Miranda y L. Toirac. "Indicadores de productividad para la industria Dominicana". Redalyc. Vol. $12 \mathrm{~N}^{\circ}$ 2, pp. 235-290. Junio 2010. ISSN: 0213-5000.

[16] M. Martínez. "Evaluación financiera y operacional aplicada en Pymes hoteleras".
Revista EAN. Vol. $65 \mathrm{~N}^{\circ}$, pp. 31-48. Febrero de 2009. ISSN: 0120-8160.

[17] L. Bortesi. "Aspectos cualitativos de la productividad". Alternativa financiera. Vol. 5 $\mathrm{N}^{\circ}$ 5, pp. 7-16. Julio 2005. ISSN: 1560-9103.

[18] H. Rincón. "Calidad, productividad y costos: Relaciones entre estos tres conceptos". Actualidad contable FACES. Vol. $4 \mathrm{~N}^{\circ} 4$, pp. 49-61 Junio de 2001. ISSN: 1316-8533.

[19] I. Alvarez, O. Becerril y L. Moral. "Efectos de la infraestructura en la productividad total de los factores y sus determinantes: un estudio en México". Estudios económicos. Vol. $26 \mathrm{~N}^{\circ}$ 1, pp. 97-122. Diciembre de 2011. ISSN: 1684-0364.

[20] A. Cuesta. "Evaluando desempeños: Alineamiento estratégico y productividad". Forum Empresarial. Vol. $17 \mathrm{~N}^{\mathrm{o}}$ 1, pp. 1-30. Junio de 2012. ISSN: 1541-8561.

[21] I. Hernández. "Forma legal, innovación y productividad en la industria manufacturera Colombiana". Cuadernos de economía. Vol. $24 \mathrm{~N}^{\circ} 42$, pp. 135-160. Abril 2005. ISSN: 0121-4772.

[22] N. Sanabria. "Investigación y Desarrollo (I+D) en la productividad". Dimension empresarial. Vol. $9 \mathrm{~N}^{\circ}$ 1, pp. 55-63. Diciembre de 2011. 0377-7332.

[23] M. Vivanco, F. Martínez y I. Taddei. Análisis de competitividad de cuatro sistema-producto estatales de tilapia en México. Estudios Sociales. Vol. 18 N$^{\circ} 2$, pp. 165-207. Diciembre 2010. ISSN: 0188-4557.

[24] M. Coronel y G. Cardona. "Tipificación de PYMES mediante técnicas de análisis multivariado: el caso de la ciudad de Santiago de Estero, Argentina". TEC empresarial. Vol. $3 \mathrm{~N}^{\mathrm{o}}$ 1, pp. 45- 54. Agosto 2009. ISSN: 1659-3359.

[25] H. Mutis. “¿Que tienen en común ciertas técnicas estadísticas multivariadas conocidas?". Revista de Ingeniería. Vol. 1 $\mathrm{N}^{\circ}$ 2, pp. 127-136. Diciembre 2003. ISSN: 2011-0049.

[26] M. Escobedo y J. Salas. "Mahalanobis y las aplicaciones de su distancia estadística". Cultura ciencia y tecnología. Vol. $5 \mathrm{~N}^{\circ} 27$, pp. 13-21. Enero 2008. ISSN: 1856-5301.

[27] B. Tabachnick and L. Fidell. "Using Multivariate Statistics". Edition No 4, pp. 104. Boston, EE.UU. 2001. ISBN: 0-321-05677-9. 
[28] M. Blanca. "Alternativas de análisis estadístico en los diseños de medidas repetidas". Psicothema. Vol. $16 \mathrm{~N}^{\circ} 3$, pp. 509-518. Octubre 2004. ISSN: 0214-9915.

[29] M. Hannula. "Total productivity measurement based on partial productivity ratios". International Journal of productions economics. Vol. $78 \mathrm{~N}^{\mathrm{o}} 1$, pp. 57-67. Febrero 2002. ISSN: 0925-5273.

[30] F. Merino. "Firms' Internationalization and productivity growth". Research in economics. Vol. $66 \mathrm{~N}^{\circ}$ 1, pp. 349-354. Agosto 2012. ISSN: 1090-9443.

[31] T. Fontalvo, A. Mendoza y J. Morelos. "Evaluación del impacto de los sistemas de gestión de la calidad en la liquidez y rentabilidad de las empresas de la Zona Industrial de Mamonal (CartagenaColombia)". Revista Católica del Norte, Vol. 1 N $^{\circ} 34$, pp. 1-28. Septiembre 2011. ISSN: 0124-5821.

[32] D. Ballesteros y P. Ballesteros. "Análisis de la productividad en el sector de las confecciones en Risaralda". Scienthia ett Technica. Vol. 12 $\mathrm{N}^{\circ} 32$, pp. 369-374. Octubre de 2006. ISSN: 0122-1701.

[33] A. Gómez. "Productividad multifactorial del sector manufacturero del Cauca. 19932006". Sociedad y Economía. Vol. 20 N$^{\circ} 1$, pp. 243-265. Enero 2010. ISSN: 1657-6357. 